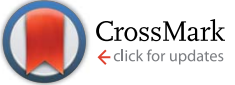

Cite this: RSC Adv., 2017, 7, 7944
Received 16th November 2016 Accepted 4th January 2017

DOI: 10.1039/c6ra26896j

www.rsc.org/advances

\section{Tribological characteristic and mechanism analysis of borate ester as a lubricant additive in different base oils}

\author{
Guangbin Yang, Junhua Zhao, Lu Cui, Shiyong Song, Shengmao Zhang, Laigui Yu \\ and Pingyu Zhang*
}

A kind of $\mathrm{N}$-containing borate ester (DEBE) with a double five-member-ring structure as a lubricant additive was synthesized by using boric acid, diethanolamine and alkylphenol polyoxyethylene ether as the starting materials. The tribological performance of the as-prepared DEBE was evaluated using a four-ball friction and wear tester in different base oils, such as liquid paraffin (LP), poly-alpha-olefin (PAO) and dioctyl sebacate (DIOS), while the morphologies of the worn scars of the steel balls were observed using a scanning electron microscope. The chemical components on the worn surfaces of the steel balls were analyzed using X-ray photoelectron spectroscopy. The tribological mechanisms in base oils of LP, PAO and DIOS were also explored. The results show that as-prepared borate ester DEBE possessed good antiwear properties in LP and PAO and can be used as a promising S- and P-free environmentally acceptable lubricating oil additive. However, the antiwear ability decreased when the additive DEBE was added to DIOS base oil. The antiwear ability of the DEBE borate esters used as an additive in DIOS might be closely related to competitive adsorption between DEBE borate ester and DIOS ester oil. Namely, compared with the DEBE additive, the DIOS base oil is first to adsorb to the metal surface because of the higher polarity. Moreover, a small amount of the additive absorbed on the metal surface may influence the continuity and compactness of the oil film formed by DIOS alone, resulting in destruction of the tribological properties of DIOS.

\section{Introduction}

Lubricants play an indispensable role in enhancing the durability and efficiency of automotive engines and industrial machinery. ${ }^{1}$ Fully formulated engine lubricants are composed of base oil and additives. It is well known that approximately $70-90 \%$ of lubricant base fluids used today are mineral oils that are derived from petroleum, and they have been widely used for more than a century. ${ }^{2}$ With the rapid development of modern industry, mechanical equipment undergoes more severe conditions like elevated temperature, high speed and heavy load, which require lubricating oils with good antiwear, loadcarrying and anti-oxidation properties. However, mineral oils can no longer satisfy the increasingly stringent demands in many specific application fields. ${ }^{3}$ More and more attention has been paid to synthetic oils, such as polyolefins (poly alpha olefin) and esters. The most important feature of these synthetic base oils is a strong antioxidant capacity, it also contains a natural detergent, so it can ensure that the lubricant is in use for a long time, and shows energy saving, environmental protection. $^{4}$ Therefore, low cost, high performance and

Engineering Research Center for Nanomaterials, Henan University, Kaifeng 475004, China.E-mail: pingyu@henu.edu.cn environmentally friendly additives for synthetic oils are urgently needed. Therefore, it is imperative to develop high-performance lubricant additives so as to guarantee the safety and reliability of various lubricants.

Over the past decades, nanoscale materials have been extensively investigated and widely employed in many scientific and technological fields due to their fascinating physical and chemical nature. ${ }^{5-7}$ And many inorganic nanoparticles have been proven to be promising as lubricant additives since they provided outstanding ability in reducing friction and wear when formulated into lubricants. ${ }^{8}$ However, inorganic nanoparticles (in particular, naked nanoclusters) have high surface activity and poor compatibility with lubricating oils and are easy to aggregate, which is usually the main obstacle to restrict their satisfactory applications.

In recent years, organic borate esters are recognized as "green" additives and have received extensive attention because of the expected antiwear (AW) and extreme pressure performance, low toxicity, and pleasant odor. ${ }^{9-11} \mathrm{~N}$-containing compounds, especially, N-containing heterocyclic compounds with compact and stable structures have recently been extensively studied. For example, benzothiazole,$^{12}$ benzotriazole,${ }^{13-15}$ quinazoline,$^{\mathbf{1 6}}$ imidazoline $^{\mathbf{1 7}}$ and triazine ${ }^{\mathbf{1 8 , 1 9}}$ groups separately introduced into the molecular backbones of borate esters and 
successfully enhanced the antiwear and friction-reducing abilities as well as load-carrying capacity of base oils. Unfortunately, most of the heterocyclic compounds involve sulfur atom which is a potential threat to the environment. Furthermore, these heterocyclic compounds are rarely applied in industry because of the high price.

Furthermore, borate esters are susceptible to hydrolysis resulting in insolubility or precipitation in oil. ${ }^{\mathbf{1 0 , 1 1}}$ The hydrolysis process is common because the electron-deficient boron element can be easily combined to water in the air. Some of the studies were focused on the introduction of some electron-rich groups into the borate esters, and the electron-deficient $\mathrm{B}$ element in the molecules can be stabilized. ${ }^{\mathbf{1 1}}$ More importantly, the introduction of nitrogen to the additive molecules, can not only enhance antiwear performance and stabilize the electrondeficient boron element because of the lone pair electrons in $p$ orbit of nitrogen which can complex the empty $2 p$ orbit of boron atom, to reduce the possibility of attack by some nucleophiles, ${ }^{20}$ but also the synergetic effect of $\mathrm{B}$ and $\mathrm{N}$ elements in borate ester additive can be found in tribological performance, the good performance of borate ester may be ascribed to the high content of $\mathrm{B}$ and $\mathrm{N}$ elements. ${ }^{\mathbf{1 1}}$ Furthermore, these $\mathrm{N}$ containing compounds are often weakly toxic and ash-free, showing great potential as a new generation of green lubricant additives. Small ${ }^{21}$ and Papay ${ }^{22}$ revealed that the hydrolytic stability of borate esters can be improved by the addition of amine compounds which is able to coordinate with electrondeficient boron to form the extra-molecular-coordination bond. By making use of the intra-molecular-coordination of nitrogen to boron, Zheng ${ }^{23}$ and $\mathrm{YaO}^{24}$ introduced imido and aminoethyl groups into borate ester molecules to form a stable six- and five-member ring structure to improve the hydrolytic stability.

In our previous research, we have tried to synthesize an $\mathrm{N}$ containing borate ester (MEBE) with five-member ring structure. ${ }^{25}$ In the present research, with the aim to improve the tribological performance and hydrolytic stability of lubricant, we select alkylphenol polyoxyethylene ether, boric acid and diethanolamine as staring materials without high price heterocyclic compounds to synthesize $S$ - and P-free DEBE borate ester with double five-member-ring structure. This article reports the details about the preparation and evaluation of the tribological properties in relation to worn surface analysis by scanning electron microscopy and X-ray photoelectron spectroscopy. And the action mechanism of as-synthesized DEBE as additive in synthetic oils (poly-alpha-olefin (PAO) and dioctyl sebacate (DIOS)) and mineral oil (liquid paraffin (LP)) was also discussed.

\section{Experimental section}

\subsection{Materials and preparation of borate ester}

Analytical grade reagents boric acid, diethanolamine and alkylphenol polyoxyethylene ether (OP-4) were provided by Tianjin Kermel Chemical Reagent Company Ltd (Tianjin, China) and Aladdin Industrial Corporation (Shanghai, China). Toluene was supplied by Luoyang Haohua Chemical Reagent Company Ltd (Luoyang, China). All the reagents were used without further purification.

A three-neck flask equipped with a mechanical stirrer, a thermometer, a reflux condenser and a Dean-Stark Trap was used to synthesize the desired borate ester. Fig. 1 shows the synthetic pathway. $50 \mathrm{~mL}$ toluene, $10 \mathrm{mmol}$ boric acid and $4.02 \mathrm{~g}$ OP-4 were added into the three-neck flask. The reactant mixture was then heated to $110-125{ }^{\circ} \mathrm{C}$ and refluxed for $3 \mathrm{~h}$ under mechanical stirring. Followed by introduction of $30 \mathrm{~mL}$ toluene and $10 \mathrm{mmol}$ diethanolamine, and refluxed there for additional $6 \mathrm{~h}$. Meanwhile, the water formed during the reaction was separated using a Dean-Stark Trap. The product was distilled under vacuum to remove toluene and water at the end of the reaction, yielding a colorless viscous liquid (the target product is denoted as DEBE), as shown in Fig. 2(a).

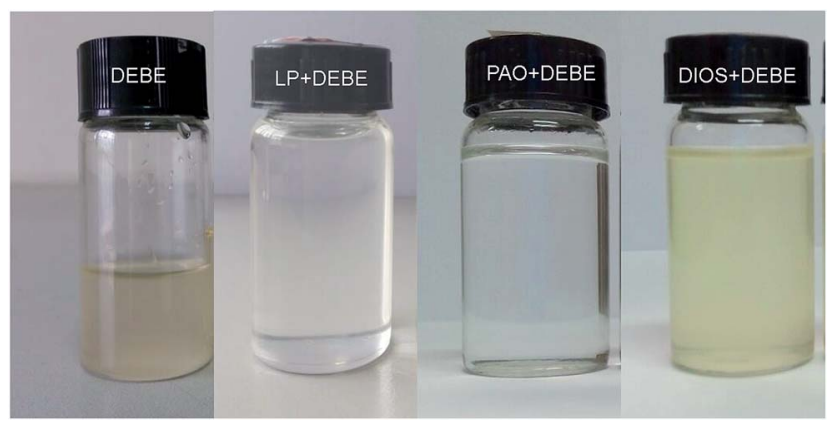

Fig. 2 Appearance of as-synthesized borate ester and optical pictures of the dispersions of as-synthesized DEBE borate ester in LP, PAO and DIOS (additive concentration: $1.0 \mathrm{wt} \%$ ).
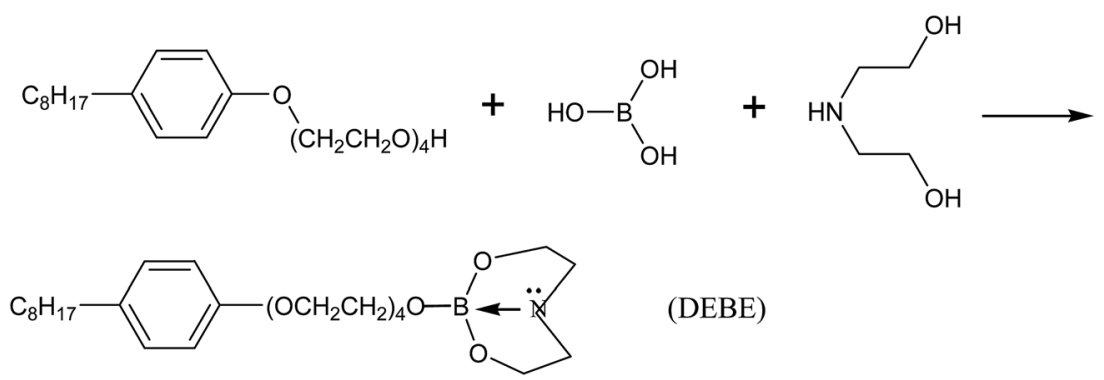

Fig. 1 Synthesis outline of additive DEBE borate ester. 


\subsection{Characterization of borate ester}

An Avatar 360 Fourier transform infrared (FT-IR) spectrometer with a resolution of $0.1 \mathrm{~cm}^{-1}$ was performed to confirm the chemical structure of as-prepared borate ester. The elements C, $\mathrm{H}$ and $\mathrm{N}$ of as-synthesized borate ester were analyzed on a Vario EL Cube elemental analyzer, with a measurement error of $<0.1 \%$. The molecular weight of borate ester was acquired on an Esquire-LC mass spectrum (MS) instrument using a direct insertion technique.

Hydrolysis stability was investigated by an open method. Borate ester with $10 \mathrm{ml} 1.0 \mathrm{wt} \%$ concentration in liquid paraffin was added to a $25 \mathrm{ml}$ dry glass, and changes in turbidity were observed. The turbidity of the liquid indicates the generation of insoluble boric acid solid. The time spent for turbidity change was used as the hydrolysis stability time.

\subsection{Tribological evaluation of borate ester}

Synthetic oil (PAO6 and DIOS) and mineral oil LP were selected as base oils without further treatment in this work. Furthermore, PAO6 and LP are alkane base oils, and DIOS is ester oil. And the typical physical parameters of the PAO6, DIOS and LP base oils are listed in Table 1.

Friction and wear tests were examined on a four ball test machine made in Jinan Testing Machine Factory of China at a rotation speed of $1450 \mathrm{rpm}$ at different loads for $30 \mathrm{~min}$ at room temperature. The maximum non-seizure loads ( $P_{\mathrm{B}}$ values) tests of additive were conducted according to the Chinese standard test method GB 3142-82, which is similar to ASTM D2783. And GCr15 bearing steel (SAE 52100 steel) balls with a diameter of $12.7 \mathrm{~mm}$ and hardness of HRc 64-66 were used to assemble the frictional pair. The wear scar diameters of the three lower balls were measured using an optical microscope with a charge-coupled device (CCD) sensor, which connects with a computer, and the friction coefficients were recorded automatically with a strain gauge attached to the test rig. At the end of each friction and wear test, the frictional pair was disassembled, and the steel balls were cleaned using petroleum ether (boiling point $60-90{ }^{\circ} \mathrm{C}$ ) in an ultrasonic bath for three times.

Then the morphologies of the wear scars on the steel balls were observed using a Nova NanoSEM450 field emission

Table 1 Typical physical properties of the three base oils

\begin{tabular}{|c|c|c|c|}
\hline \multirow[b]{2}{*}{ Parameter } & \multicolumn{3}{|c|}{ Base oils } \\
\hline & LP & PAO6 & DIOS \\
\hline Density $\left(15^{\circ} \mathrm{C}, \mathrm{g} \mathrm{cm}^{-3}\right)$ & 0.8465 & 0.819 & 0.9119 \\
\hline \multicolumn{4}{|l|}{ Kinematic viscosity $\left(\mathrm{mm}^{2} \mathrm{~s}^{-1}\right)$} \\
\hline $40{ }^{\circ} \mathrm{C}$ & 21.49 & 31 & 23.4 \\
\hline $100{ }^{\circ} \mathrm{C}$ & 4.42 & 5.8 & 5.2 \\
\hline Viscosity index & 117 & 138 & 162 \\
\hline Pour point $\left({ }^{\circ} \mathrm{C}\right)$ & -15 & -68 & -41 \\
\hline Flash point $\left({ }^{\circ} \mathrm{C}\right)$ & 226.5 & 246 & 210 \\
\hline Total acid number, $\mathrm{mgKOH} \mathrm{g}^{-1}$ & $\leq 0.05$ & $\leq 0.03$ & $\leq 0.21$ \\
\hline
\end{tabular}

scanning electron microscope (FESEM) equipped with an energy dispersive spectrometer (EDS) accessory. And X-ray photoelectron spectroscopy (XPS, Kratos Axis-Ultra) was used to analyze some typical elements on the worn surfaces of the steel balls.

\section{Results and discussion}

\subsection{Characterization of as-synthesized borate ester}

The product was characterized by means of Fourier transform infrared (FT-IR) spectrometer, elemental analyzer and mass spectrum. The FT-IR spectrum of the as-synthesized borate ester DEBE was shown in Fig. 3. The significant features of $\mathrm{N}$ containing borate ester are the bands corresponding to $\mathrm{N}-\mathrm{H}$ stretching vibration at $3400 \mathrm{~cm}^{-1}$, and the peak at $1247 \mathrm{~cm}^{-1}$ corresponds to $\mathrm{C}-\mathrm{N}-\mathrm{C}$ bonds. ${ }^{26}$ It is known that for borate ester molecule containing $\mathrm{B}-\mathrm{O}$ bonds, and $\mathrm{B}-\mathrm{O}$ infrared absorption peaks appear at wave numbers of $1430-1355 \mathrm{~cm}^{-1}$. It can be seen from Fig. 3 that a strong $\mathrm{B}-\mathrm{O}$ infrared absorption peak appears at $1358 \mathrm{~cm}^{-1} \cdot{ }^{27,28}$ And $1108 \mathrm{~cm}^{-1}$ is the peak of $\mathrm{C}-\mathrm{O}$ stretching vibration in borate ester, it is one of characteristic absorption peaks of borate ester. ${ }^{28}$ Besides, Benzene ring skeletal vibration is at about 1611,1510 and $1459 \mathrm{~cm}^{-1}$. The absorption bands at near $2958 \mathrm{~cm}^{-1}$ and $2873 \mathrm{~cm}^{-1}$ are assigned to the asymmetric and symmetric $-\mathrm{CH}_{2}$ stretch vibrations. This was further supported by the corresponding mass specturm and element analysis results. The results listed in Table 2 are in agreement with the calculated ones, primarily indicating that the synthesized compound is the target product.

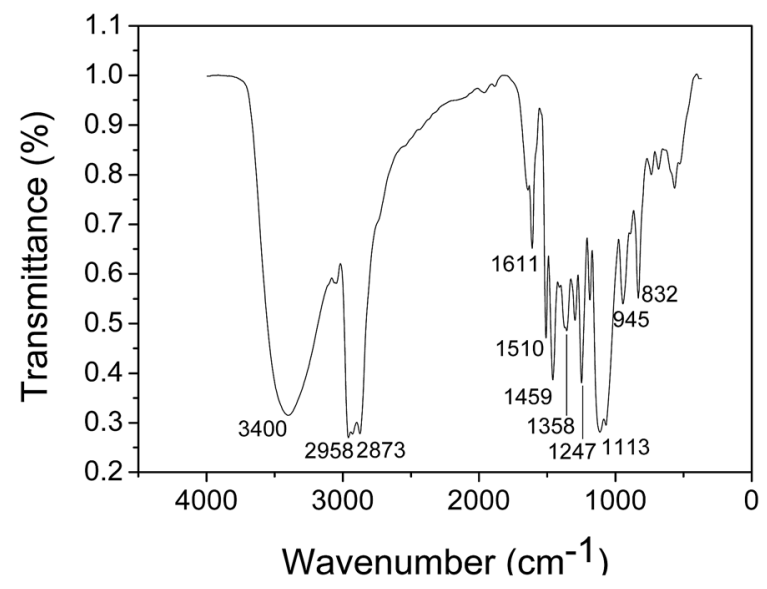

Fig. 3 FT-IR spectrum of the as-synthesized DEBE borate ester.

Table 2 Element analysis and mass spectrum data of the as-synthesized DEBE borate ester

\begin{tabular}{llllll}
\hline & & \multicolumn{3}{c}{ Composition (\%) } \\
\cline { 3 - 5 } Products & Molecular weight & $\mathrm{C}$ & $\mathrm{H}$ & $\mathrm{N}$ \\
\hline DEBE-experiment & 494 & 63.74 & 9.30 & 2.53 \\
DEBE-theoretical & 494.44 & 63.15 & 9.17 & 2.83
\end{tabular}


The uniform dispersion of DEBE borate ester for $0.6 \mathrm{wt} \%$ in LP, PAO and DIOS is shown in Fig. 2(b)-(d), respectively. Fig. 2(b) and (c) appear colorless and transparent without clouds or phase separation by visual inspection at room temperature. And Fig. 2(d) shows transparent, light yellow, and no precipitate was observed at the bottom for a week under ambient conditions. It indicates that the as-synthesized borate ester has good dispersibility in three kinds of base oils.

The turbidity of petroleum wax oil did not change after 1 month at room temperature when $1.0 \mathrm{wt} \%$ DEBE was added, which is much longer than the turbidity time of trimethyl borate ( $5 \mathrm{~min}$ ) and tributhyl borate $(10 \mathrm{~min})$ in the literature. ${ }^{20,28}$ The results illustrated that DEBE borate ester possessed excellent hydrolytic stability. This is mainly because the oxygen and nitrogen can be helpful for stabilizing the deficient B element in the molecule. ${ }^{10}$ On the other hand, the nitrogen in DEBE can form five-member-ring intra-molecular-coordination, which is proved to be stable. ${ }^{23,24}$

\subsection{Tribological performance of as-synthesized borate ester}

Fig. 4 depicts the relationship between friction coefficient and additive concentration in LP, PAO and DIOS base oils under the applied load of $200 \mathrm{~N}$ (corresponding to the maximum Hertz Contact Pressure is $2664 \mathrm{MPa}){ }^{25}$ It can be seen from the curves that the friction coefficient decreased slightly when DEBE borate ester was introduced into DIOS. And the friction coefficient sometimes was even a little higher than the one under the lubrication of LP alone, as the additive concentration in LP increased, Furthermore, under the same conditions, the friction coefficient of DEBE in liquid paraffin is lower than that of MEBE borate ester in liquid paraffin which is we synthesized in the past. $^{25}$ However, the friction coefficient is low and almost unchanged with the increase additive concentration in PAO. In general, it was an interesting phenomenon that the friction coefficient did not change obviously compared with the base oils, which suggests that there is no high shearing strength component generated on the surface of the frictional pairs. ${ }^{29} \mathrm{It}$ is generally accepted that the friction-reducing property is

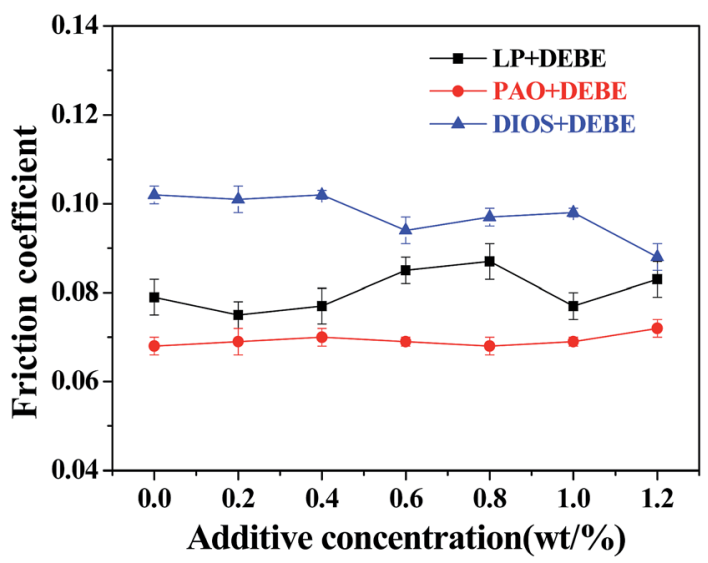

Fig. 4 Variation of friction coefficient with additive concentration in different based oil (four-ball tester; load: $200 \mathrm{~N}$, speed: $1450 \mathrm{rpm}$, duration: $30 \mathrm{~min}$ ). pertinent to the shearing strength and low shearing strength corresponds to low friction coefficient. DEBE did not generate high shearing strength component which results in stable friction coefficient.

Fig. 5 illustrates the variation of wear scar diameter of the three lower steel balls with additive concentration in LP, PAO and DIOS base oils (four-ball tester; load: $200 \mathrm{~N}$, speed: $1450 \mathrm{rpm}$, duration: $30 \mathrm{~min}$ ). It can be seen that the introduction of DEBE in LP leads to a sharply decrease of WSD when the concentration is less than $0.4 \%$. When the concentration of DEBE additive is within $0.4-1.0 \%$, the WSD varies slightly with increasing additive concentration. And the smallest wear scar diameter of $0.37 \mathrm{~mm}$ was obtained at a concentration of $0.8 \%$ in LP, which was reduced by $30.8 \%$ as compared with that under the lubrication of LP alone $(0.52 \mathrm{~mm})$. However, the WSD increases with further increase of additive concentration above $1.0 \%$. Furthermore, compared with MEBE borate synthesized in the past,${ }^{25}$ the antiwear property of DEBE was superior to that of MEME at low additive concentration (less than $0.6 \%$ in LP). However, when the additive concentration was more than $0.6 \%$, DEBE is lower in antiwear performance than MEBE. The difference is supposed to be related to their polarity and molecular structure which affect the adsorption ability and the reaction activity. ${ }^{\mathbf{1 1 , 3 0}}$ As the DEBE additive was added into PAO base oil, the WSD decreases obviously with increasing concentration of additive DEBE, and the minimum wear scar diameter of $0.38 \mathrm{~mm}$ was recorded at an additive concentration of 0.6 $\mathrm{wt} \%$, which corresponds to a reduction by $23.9 \%$. However, above $0.6 \mathrm{wt} \%$, the wear scar diameter tends to increase with further increase of the additive concentration up to $1.2 \%$. The reason for the increasing antiwear ability of LP and PAO lies in that the DEBE additive is easy to enter into the rubbing surface and be deposited thereon to form a surface protective film under local high temperature and high contact pressure, thereby preventing the direct contact of the rubbing surfaces and greatly reducing wear. Above the optimum concentration, the wear scar diameter tended to slightly increase with further increase of the concentration, which might be due to the fact that excessive additives were delivered to the contact area and

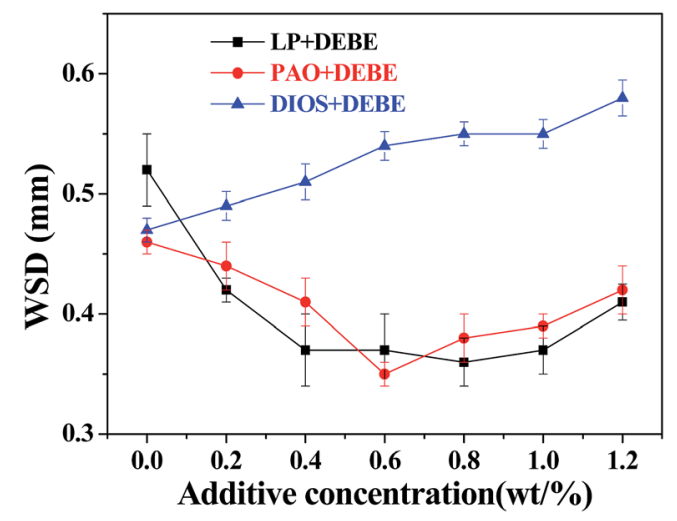

Fig. 5 Variation of wear scar diameter with additive concentration in different based oils (four-ball tester; load: $200 \mathrm{~N}$, speed: 1450 rpm, duration: $30 \mathrm{~min}$ ). 
lead to coagulation at the interface of rubbing-pair. This in turn causes the increase of friction force and unstable operation, even the local damage of tribofilm, and thus results in higher wear in the presence of too much additive. ${ }^{31}$

However, when the additive DEBE borate ester was introduced into DIOS base oil, the wear scar diameter increased slightly with increasing additive concentration in the test concentration range. As-synthesized borate ester has negative impact on the antiwear ability of DIOS, which is supposed to be related to the competitive adsorption between DEBE and DIOS. It is well known that DIOS has high polarity and can reduce friction and wear by generating a relatively thick and low shear stress oil film with the assistance of chemical adsorption. Meanwhile, the synthesized additive also has polarity. So according to the test results, DIOS has higher polarity than DEBE which affects the adsorption ability and the reaction activity. ${ }^{11,32}$ DIOS is preferentially adsorbed onto the metal surface, and becomes the dominant adsorbates, generating a stable tribofilm on the steel ball surface when the additive concentration is low. Nevertheless, the additive may influence the compactness and continuity of the oil film formed by the DIOS alone with the increasing of additive concentration, so the antiwear ability became weaker than that of DIOS alone.

The plots of wear scar diameter as a function of applied load at 30 min test duration were shown in Fig. 6 . It can be seen that the WSDs were very large in the absence of additive but in the presence of the additive it is fairly reduced under selected loads, when the DEBE additive was added into LP and PAO base oils, respectively (Fig. 6(a) and (b)). This indicates that DEBE borate ester can reduce the wear scar diameter of LP and PAO base oils at applied load range from 100 to $400 \mathrm{~N}$. the decreases in WSD can be attributed to the fact that there is a strong attraction between electron-deficient $\mathrm{B}$ atom and metallic surface because $B$ atom with vacant p-orbital can capture the electrons of the d- or f-orbital and free electrons of metallic balls, promoting the adsorption of borate ester onto the metallic surface and the formation of a dense adsorbed film, which can avoid the direct contact between the sliding steel surfaces, resulting in reducing wear of the steel-steel sliding pair. ${ }^{32}$ But, the additive introduced into DIOS did not enhance the antiwear performance of the base oil under the test loads (Fig. 6(c)). This also could be ascribed to DIOS with higher polarity than DEBE which affects the adsorption ability, the reaction activity ${ }^{11,30}$ as well as continuity of the oil film formed by the DIOS alone. Furthermore, it is evident that WSD rises with the increasing of normal load in the three kinds of base oils. This may be due to the fact that the film thickness formed between the contact area decreases with the increasing of normal load, resulting in the increasing of direct contact of asperities, and then WSD rises. ${ }^{\mathbf{1 0}}$

The extreme pressure performance of DEBE was evaluated as the maximum non-seizure load $\left(P_{\mathrm{B}}\right)$ in three based oils, and the results are shown in Fig. 7 (four-ball tester; speed: $1450 \mathrm{rpm}$, duration: $10 \mathrm{~s}$ ). It can be noted that $P_{\mathrm{B}}$ value is slightly enhanced when DEBE was added into the three based oils, which is related to the formation of protective film on the friction surface.
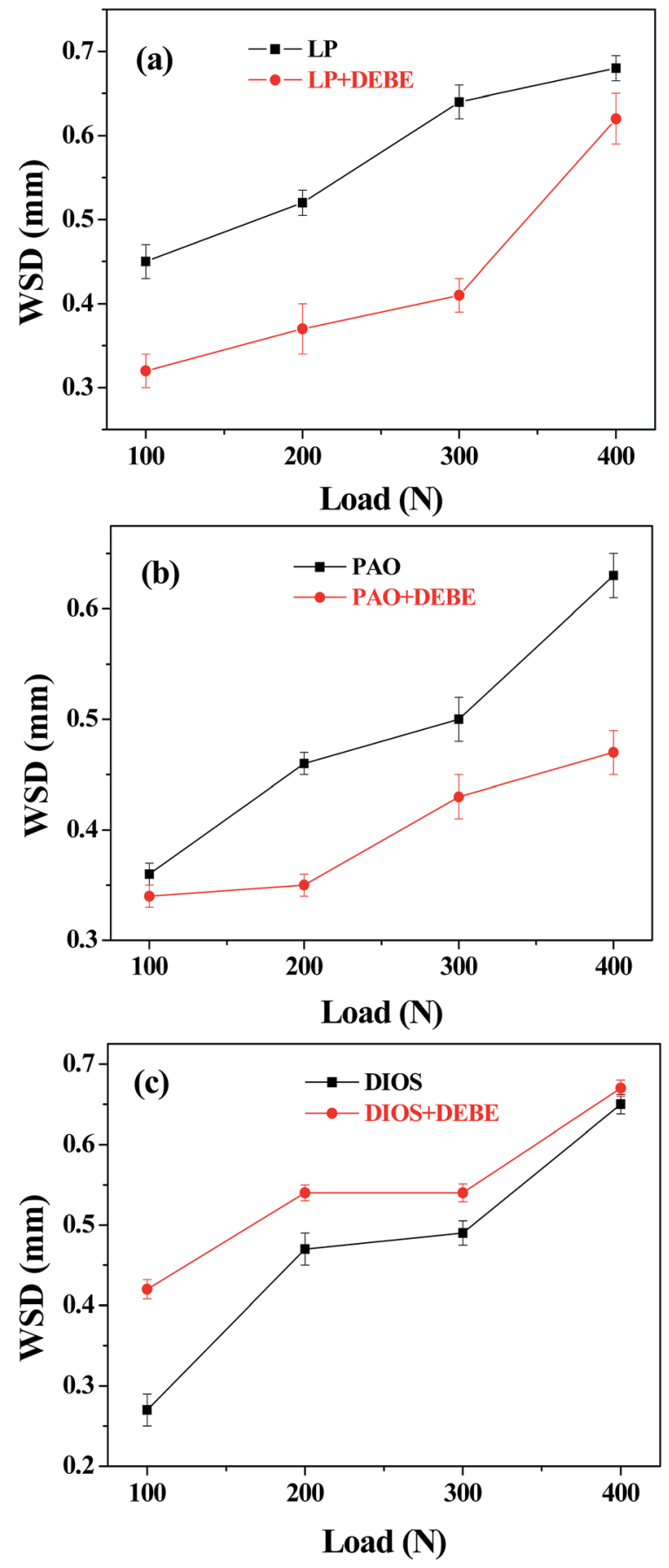

Fig. 6 Variation of wear scar diameter with applied load, additive in (a) LP, (b) PAO and (c) DIOS (four-ball tester; speed: 1450 rpm, duration: $30 \mathrm{~min})$.

\subsection{Mechanism analysis of as-synthesized borate ester}

In order to understand the action mechanism of additive in friction process, the worn surface of steel ball was studied by SEM in this work. Fig. 8 displays the SEM images of worn surfaces of steel balls lubricated by LP, PAO and DISO with or without DEBE borate ester. In the case of the steel surface lubricated with base oil LP (Fig. 8(a) and (b)) and PAO (Fig. 8(e) and (f)) huge surface destruction with severe deformation is 


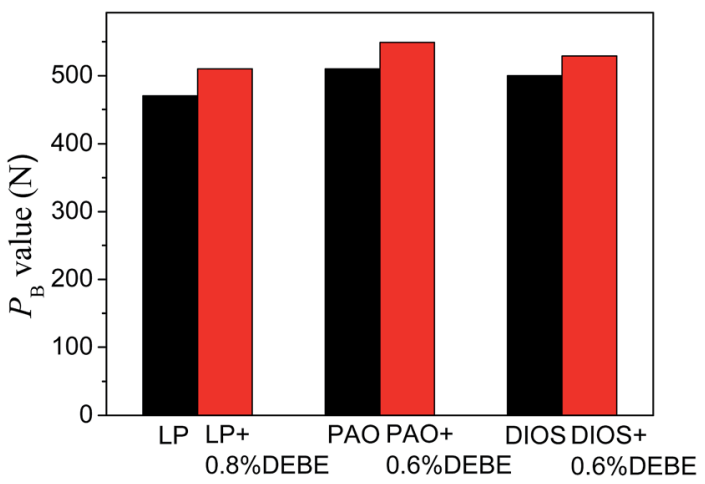

Fig. 7 Maximum non-seizure loads ( $P_{\mathrm{B}}$ values) of the as-synthesized DEBE in the three based oils (four-ball tester, rotation velocity $1450 \mathrm{rpm}, 10 \mathrm{~s})$.

observed due to the grain abrasion wear, indicating that severe wear and scuffing occurs during the test, which is ascribed to the poor lubricating film strength formed by base oil alone. On the other hand, the worn surfaces in the presence of additive in LP and PAO are smoother and no sign of severe scuffing along the sliding direction, and the wear scar are relatively narrow and shallow (Fig. 8(c), (d), (g) and (h)) in comparison to the surface lubricated with base oil alone. This observation results indicated that the as-synthesized DEBE borate ester could reduce wear of the steel balls to some extent when it was separately introduced into LP and PAO, well corresponding to corresponding friction and wear test data presented in Fig. 5 and 6. On the contrary, when the DEBE additive was introduced into DIOS, the WSD is slight larger than that lubricated by base oil DIOS alone (Fig. 8(i) and (j)), the worn surface lubricated by DIOS-DEBE (Fig. 8(k) and (l)) also shows obvious deep furrows along the sliding direction like that by DIOS alone. This confirms that the additive in DIOS could not be effective in reducing wear of the steel-steel friction pairs.

The element composition of the worn surfaces lubricated by LP, PAO and DIOS with and without DEBE obtained from EDS analysis was listed in Table 3 . It can be seen that the elements on the wear scar were $\mathrm{Fe}, \mathrm{Cr}, \mathrm{O}$ and $\mathrm{C}$ when the steel ball was lubricated with LP, PAO and DIOS alone, respectively. Besides the above mentioned elements, $\mathrm{B}$ and $\mathrm{N}$ elements were also detected by EDS on the worn surface of the steel ball, which may derive from additive DEBE when it as additive was added in LP
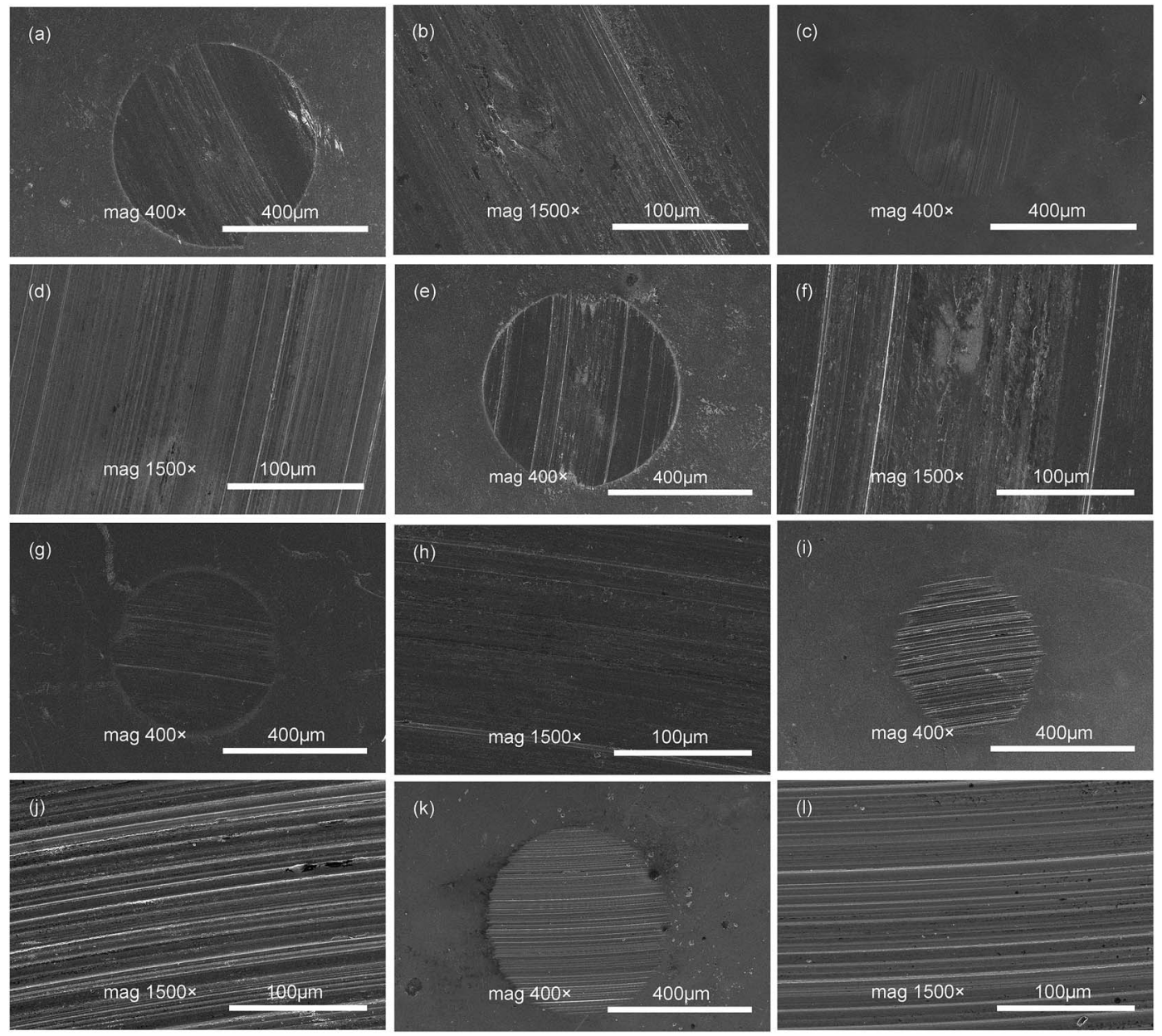

Fig. 8 SEM pictures of worn steel surfaces lubricated with (a and b) LP alone, (c and d) LP containing $0.8 \%$ DEBE, (e and f) PAO alone, (g and $h$ ) PAO containing $0.6 \%$ DEBE, ( $\mathrm{i}$ and $\mathrm{j}$ ) DIOS alone, and ( $\mathrm{k}$ and l) DIOS containing $0.6 \%$ DEBE (four-ball tester; load: $200 \mathrm{~N}$, speed: 1450 rpm, duration: $30 \mathrm{~min})$. 
Table 3 Elemental composition of worn steel surface lubricated with LP, PAO and DIOS with and without the as-synthesized borate esters, respectively

\begin{tabular}{lllllll}
\hline & \multicolumn{7}{l}{ Elemental composition (wt\%) } \\
\cline { 2 - 7 } Lubricant & $\mathrm{B}$ & $\mathrm{C}$ & $\mathrm{N}$ & $\mathrm{O}$ & $\mathrm{Cr}$ & $\mathrm{Fe}$ \\
\hline LP & 0 & 4.31 & 0 & 1.81 & 2.01 & 91.87 \\
LP - 0.8\% DEBE & 2.87 & 3.73 & 0.45 & 1.5 & 1.34 & 90.11 \\
PAO & 0 & 9.93 & 0 & 8.87 & 1.52 & 79.68 \\
PAO - 0.6\% DEBE & 2.12 & 5.76 & 2.04 & 7.45 & 1.32 & 81.31 \\
DIOS & 0 & 10.47 & 0 & 6.21 & 1.55 & 81.77 \\
DIOS - 0.6\% DEBE & 0 & 10.08 & 1.01 & 4.42 & 1.26 & 83.23 \\
& & & & & &
\end{tabular}

and PAO, respectively. The test results proved that assynthesized additive is indeed deposited on worn steel surfaces through adsorption and/or tribochemical reaction, thereby forming a mixed boundary protective tribofilm thereby reducing wear of the steel-steel sliding pairs. But B element was not detected by EDS on the wear scar of the steel ball lubricated by DIOS containing DEBE additive. This result revealed that $\mathrm{B}$ element may not be adsorbed onto the surface of the metal ball.

XPS analysis of the worn surface is performed to ascertain the chemical compositions of tribofilm and explore possible tribofilm forming mechanisms of DEBE as additive in different base oils. Fig. 9 shows the XPS spectra of B1s, N1s, O1s, C1s and $\mathrm{Fe} 2 \mathrm{p}$ on the worn steel surface lubricated with LP containing $0.8 \%$ DEBE additive at $200 \mathrm{~N}$ load for $30 \mathrm{~min}$ test duration. The $\mathrm{B} 1 \mathrm{~s}$ peaks at $192.0 \mathrm{eV}$ and $\mathrm{O} 1 \mathrm{~s}$ at $532.4 \mathrm{eV}$ are assigned to $\mathrm{B}_{2} \mathrm{O}_{3},{ }^{10,29,33}$ and it suggests that the DEBE additive was degraded and reacted with oxygen on the metal surface during the friction process. The N1s peak located at around $399.9 \mathrm{eV}$ is corresponded to organic $\mathrm{N}$ of ethanolamine, ${ }^{28}$ suggesting that $\mathrm{N}$ in the form of organic $\mathrm{N}$-containing compound was adsorbed on
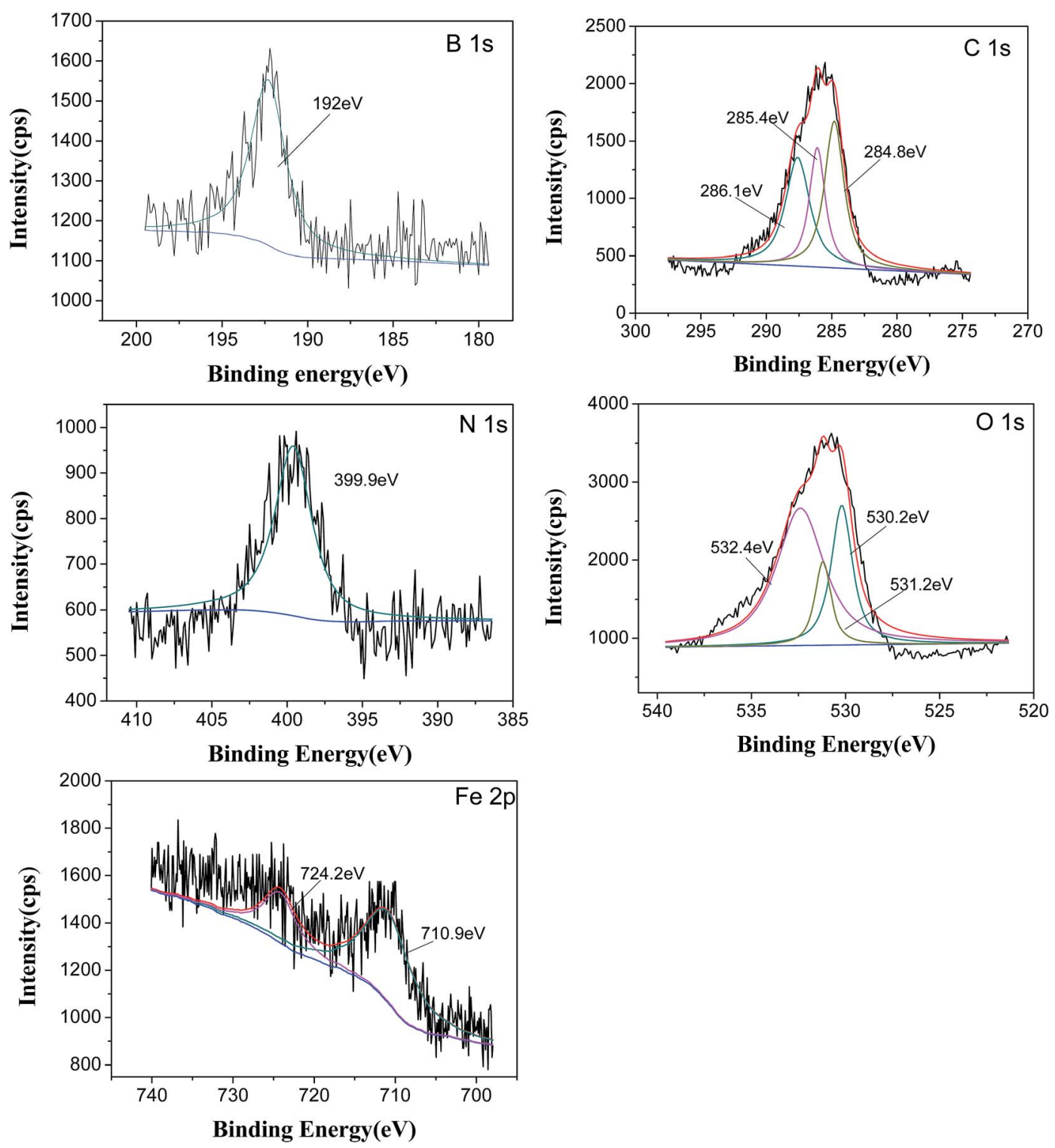

Fig. 9 XPS spectra of worn steel surfaces lubricated with LP + 0.8\% DEBE (four-ball tester; load: 200 N, speed: 1450 rpm, duration: 30 min). 
the steel ball surface. In case of $\mathrm{C}$ element, the C1s peaks at 284.8, 285.4 and $286.1 \mathrm{eV}$ are attributed to $\mathrm{C}-\mathrm{H}, \mathrm{C}-\mathrm{C}$ and $\mathrm{C}-\mathrm{O}$ bonds in organic compounds, ${ }^{26,33}$ which suggest that the decomposed products of DEBE additive were adsorbed on the metal surface. Oxidation of iron to $\mathrm{Fe}_{2} \mathrm{O}_{3}$ during the rubbing process has been confirmed by combining the binding energies of Fe2p at $710.9 \mathrm{eV}$ with $\mathrm{O} 1 \mathrm{~s}$ at $530.2 \mathrm{eV},{ }^{34}$ which is ascribed to the tribochemical reaction on the friction pairs. The O1s peaks at $530.2 \mathrm{eV}, 531.2 \mathrm{eV}$ and 532.4 correspond to $\mathrm{Fe}_{2} \mathrm{O}_{3}, \mathrm{C}-\mathrm{O}$ bond of OP-4 group ${ }^{35}$ and $\mathrm{B}_{2} \mathrm{O}_{3}$, respectively. In addition, the XPS spectra on the worn steel surface lubricated by PAO-DEBE are similar to that by LP-DEBE. These XPS results reveal that DEBE additive in LP or PAO was degraded first on the steel surface, then took place complicated tribochemical reaction on the friction pairs during the test and generated a protective film on the rubbing surface. The inorganic and organic protective films composing of $\mathrm{B}_{2} \mathrm{O}_{3}, \mathrm{Fe}_{2} \mathrm{O}_{3}$, polyoxyethylene ether moiety and other N-containing organic compounds, effectively improved the antiwear properties of PAO and LP, respectively.

The XPS spectra of B1s, N1s, C1s O1s, and Fe2p of the worn surface lubricated with DIOS - 0.6\% DEBE at $200 \mathrm{~N}$ for $30 \mathrm{~min}$ are shown in Fig. 10. It is interesting to note that B1s peak is not observed in the spectrum showing that boron was not absorbed onto the worn surface, or was not enough to detect by XPS, which was well in agreement with the corresponding EDS data presented in Table 3. As for the $\mathrm{N}$ element on the worn surface, the binding energy of N1s at $399.9 \mathrm{eV}$ can be assigned to the adsorbed organic nitrogen of ethanolamine. ${ }^{28}$ Furthermore, combining the binding energies of O1s at $530.1 \mathrm{eV}$ with Fe2p at $711.3 \mathrm{eV}$, it can be stated that steel surface is liable to oxidize to form $\mathrm{Fe}_{2} \mathrm{O}_{3}$ during the friction process. These XPS results indicate that the additive could not be adsorbed much onto the metal surface and almost no tribochemical reactions occurred with other elements. It implies that different mechanisms
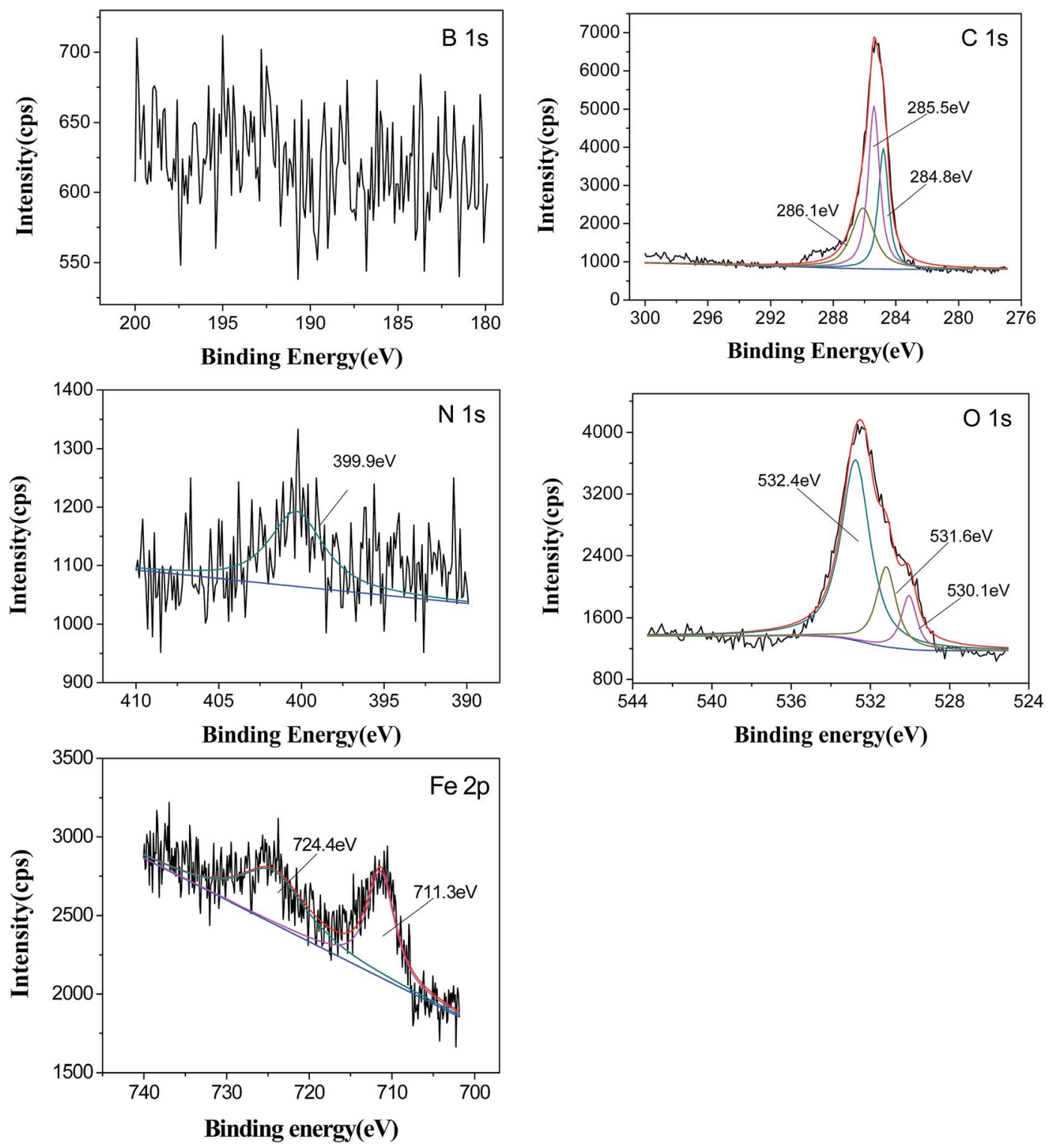

Fig. 10 XPS spectra of worn steel surfaces lubricated with DIOS + 0.6\% DEBE (four-ball tester; load: 200 N, speed: 1450 rpm, duration: 30 min). 


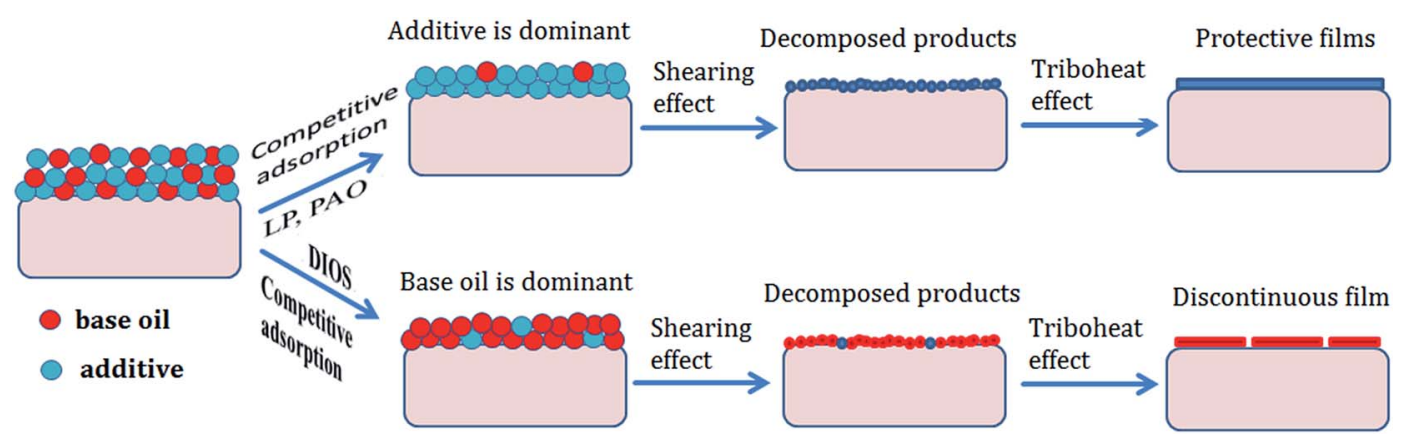

Fig. 11 Schematic diagram of the action mechanism of as-synthesized borate ester in different base oils.

might occur in the different base oils. This is also due to the competitive adsorption between DISO and DEBE for their polar groups. As we know, the order of polarity of functional groups is $-\mathrm{CH}_{3}<-\mathrm{OCOR}<-\mathrm{NH}_{2}<-\mathrm{OH}<-\mathrm{COOH}$. Decomposed product of $\mathrm{N}$-containing organic compound can be adsorbed and enriched on the worn surface because it is higher polarity than the ester oil.

According to the above results, the action mechanism of DEBE borate ester as additive in different base oils was shown in Fig. 11.

First, based on the polarity and reaction activity, there exists a competitive adsorption between the DEBE additive and base oil (LP, PAO or DIOS) in the rubbing process. The DEBE can be adsorbed onto the metal surface to form an adsorption film (physical adsorption) when the polarity of DEBE additive is higher than that of base oil (LP or PAO). And vice versa, the base oil DIOS was adsorbed and became the dominant adsorbate on the steel ball surface.

Subsequently, the adsorbed additive on the metal surface decomposed under shearing effect, high temperature and high pressure during rubbing process. Some decomposed products were directly adsorbed on the metal surface to form adsorbed film (physical and chemical adsorption), and others took place tribochemical reaction with other active elements under high triboheat to produce protective tribofilm. For example, adsorbed DEBE additive molecule in LP and PAO decomposed on metal surface, ethanolamine group and alkylphenol polyoxyethylene ether moiety were adsorbed on the worn surface, and the borate group reacted with the metal surface to form inorganic boron oxygen film, which possessed a high degree of hardness, contributing to improve antiwear performance as reported in other literatures. ${ }^{20,31,32}$ The protective film composing of $\mathrm{B}_{2} \mathrm{O}_{3}$, $\mathrm{Fe}_{2} \mathrm{O}_{3}$, N-containing organic compounds and other alkylphenol polyoxyethylene ether moiety could effectively prevent the rubbing surfaces from direct contact, thereby retarding adhesion wear, which also accounts for increased antiwear ability.

However, the DEBE additive has a negative effect on the antiwear ability when the polarity of base oil is high because a small amount of DEBE additive absorbed on the metal surface may destroy the continuity and compactness of the oil film formed by the DIOS alone. The compactness and roughness of the oil film were related to the AW and friction-reducing properties. ${ }^{36} \mathrm{~A}$ non-compact and discontinuous oil film led to deterioration of the wear resistance.
Therefore, first of all, additives should be compatible with the base oil. Then a dense and smooth protective tribofilm could be formed on the worn steel surface, which could lead to improve the tribological properties of the base oil.

\section{Conclusions}

The tribological properties and action mechanism of DEBE borate ester as additive in different base oils have been investigation and discussed. The results can be summarized as follows:

(1) A kind of N-containing borate ester DEBE without S and P elements was successfully synthesized and characterized, which could meet the requirement of environmental protection.

(2) When the additive DEBE was introduced into LP or PAO, the additive molecular adsorbed on the metal surface decomposes and reacts with metal surface and other active elements during the friction process to form a compact protective film on the rubbed surface. The resulting protective film consisting of $\mathrm{B}_{2} \mathrm{O}_{3}, \mathrm{Fe}_{2} \mathrm{O}_{3}$, polyoxyethylene ether moiety and other $\mathrm{N}$ containing organic compounds contributed to the improving the antiwear ability of the base stock LP and PAO.

(3) As-synthesized borate ester DEBE as lubricant additive in DIOS led to decrease the antiwear properties. This is mainly because there is competition adsorption between borate ester and ester oil DIOS. DIOS adsorption capacity was much more than DEBE on metal surface because of its high polarity, and smaller amount of adsorption DEBE in friction surface, resulting in DIOS base oil continuous lubricating film not be formed on the metal surface, the final tribological properties decrease.

\section{Acknowledgements}

The authors thank the financial supports from the Ministry of Science and Technology of China (project of "973" Plan, grant No. 2013CB632303) and the National Natural Science Foundation of China (grant No. 51275154 and 51375142).

\section{References}

1 R. Wright, K. Wang, J. Qu and B. Zhao, Angew. Chem., Int. Ed., 2016, 55, 8656-8660.

2 Y. Wu, W. Li and X. Wang, Lubr. Sci., 2015, 27, 369-379.

3 W. J. Bartz, Tribol. Int., 2006, 39, 728-733. 
4 I. Madanhire and C. Mbohwa, Synthetic lubricants and the environment, in Mitigating environmental impact of petroleum lubricants, ed. I. Madanhire and C. Mbohwa, Springer International Publishing, Switzerland, 2016, pp. 59-72.

5 B. Chen, K. Gu, J. Fang, J. Wu, J. Wang and N. Zhang, Appl. Surf. Sci., 2015, 353, 326-332.

6 D. L. Jiang, X. L. Hu, R. Wang and D. Q. Yin, Chemosphere, 2015, 122, 8-13.

7 V. Ya and S. L. Rudyak, Phys. Lett. A, 2014, 378, 1845-1849.

8 Z. Tang and S. Li, Curr. Opin. Solid State Mater. Sci., 2014, 18, 119-139.

9 F. U. Shah, S. Glavatskih and O. N. Antzutkin, Tribol. Lett., 2013, 51, 281-301.

10 J. C. Yan, X. Q. Zeng, E. Heide, T. H. Ren and Y. D. Zhao, RSC Adv., 2014, 4, 20940-20947.

11 J. Yan, X. Zeng, E. Heide and T. Ren, Tribol. Int., 2014, 71, 149-157.

12 J. S. Li, X. H. Xu, Y. G. Wang and T. H. Ren, Tribol. Int., 2010, 43, 1048-1053.

13 J. Li, Y. Zhang, T. Ren, W. Liu and X. Fu, Wear, 2002, 253, 720-724.

14 B. Yu, F. Zhou, C. Pang, B. Wang, Y. Liang and W. Liu, Tribol. Int., 2008, 41, 797-801.

15 Z. Jia, Y. Xia, X. Pang and J. Hao, Tribol. Lett., 2011, 41, 247256.

16 P. Ouyang, G. X. Chen, H. F. Li and L. T. Zhao, Lubr. Sci., 2010, 22, 209-214.

17 Q. Xue, J. Zhang, W. Liu and S. Yang, Tribol. Lett., 1999, 7, 27-30.

18 R. Qiao, J. Li, H. Wu, T. Ren, Y. Zhao and C. Ma, Appl. Surf. Sci., 2011, 257, 3843-3849.

19 J. Li, B. Fan, T. Ren and Y. Zhao, Tribol. Int., 2015, 88, 1-7.
20 L. Xiong, Z. He, L. Qian, L. Mu, A. Chen, S. Han, J. Qiu and X. Fu, PLoS One, 2014, 9, e83501-e83509.

21 V. R. Small and T. V. Liston, USP 4975211, 1990.

22 A. G. Papay and J. P. Brien, USP 4295983, 1981.

23 Z. Zheng, G. Q. Shen, Y. Wan, L. L. Cao, X. D. Xu and Q. X. Yue, Wear, 1998, 222, 135-144.

24 J. B. Yao, Q. L. Wang, J. X. Dong, S. Q. Chen and J. Z. Sun, Lubr. Sci., 2002, 14, 415-423.

25 F. Jin, G. Yang, Y. Peng, S. Zhang, L. Yu and P. Zhang, Lubr. Sci., 2016, 28, 505-519.

26 G. B. Yang, J. F. Zhang, S. M. Zhang, L. G. Yu, P. Y. Zhang and B. L. Zhu, Tribol. Int., 2013, 62, 163-170.

27 J. C. Li, Z. P. Li, T. H. Ren, X. Q. Zeng and E. V. Heide, Tribol. Int., 2014, 73, 101-107.

28 Z. Y. He, L. P. Xiong, L. Qian, S. Han, A. X. Chen, J. W. Qiu and X. S. Fu, Lubr. Sci., 2014, 26, 81-94.

29 Z. Li, X. Li, Y. Zhang, T. Ren, Y. Zhao, X. Zeng and E. Heide, Appl. Surf. Sci., 2014, 308, 91-99.

30 Y. W. Zhang, X. Q. Zeng, H. Wu, Z. P. Li, T. H. Ren and Y. D. Zhao, Tribol. Lett., 2014, 53, 533-542.

31 S. Wang, W. Yue, Z. Q. Fu, C. B. Wang, X. L. Li and J. J Liu, Tribol. Int., 2013, 66, 259-264.

32 G. B. Yang, Z. M. Zhang, G. H. Li, J. F. Zhang, L. G. Yu and P. Y. Zhang, Trans. ASME, J. Tribol., 2011, 133, 021801021807.

33 J. Yan, X. Zeng, T. Ren and E. Heide, Appl. Surf. Sci., 2014, 315, 415-424.

34 K. Kalkhanday, V. Jaiswal, R. B. Rastogi and D. Kumar, $R S C$ Adv., 2014, 4, 30500-30510.

35 W. Li, Y. Wu, X. Wang and W. Liu, Lubr. Sci., 2011, 23, 363373.

36 Z. P. Li, Y. L. Li, Y. W. Zhang, T. H. Ren and Y. D. Zhao, RSC Adv., 2014, 4, 25118-25126. 